\title{
Diversity and Training Delivery Trends in Psychological First Aid: Implications for Educators and Researchers
}

\author{
Chung-Fan Ni ${ }^{1}$ (D) https://orcid.org/0000-0002-7881-5985 \\ Robert Lundblad ${ }^{2}$ (D) https://orcid.org/0000-0002-7520-89 \\ Cass Dykeman $^{3}$ (D) https://orcid.org/0000-0001-7708-1409 \\ ${ }^{1}$ Western Oregon University \\ ${ }^{2}$ Bolante.net \\ ${ }^{3}$ Oregon State University
}

This is the authors' preprint. All comments are welcome and can be directed to the second author at Robert.Lundblad@Bolante.net

\begin{abstract}
Psychological First Aid (PFA) refers to evidence-supported intervention by non-mental health professionals to assist those affected by disaster to re-achieve stability through a stepwise process (Ruzek et al., 2007). This study reviewed the academic discourse regarding PFA through all available abstracts in the Web of Science database from 1975 to 2021with the keyword searches for psychological first aid. The corpus linguistic analyses using \#Lancsbox 6.0 and Sketch Engine explored the usage rate of psychosocial first aid and how the PFA was used. The study also examined race/ethnicity, learning delivery except for online, and online training delivery methods within the PFA academic articles. The change of online PFA training delivery with the advent of the COVID-19 pandemic was analyzed using Tau. The study found a substantive discourse on PFA service and delivery but little on race and ethnicity. There was a significant increase in PFA online training since COVID19 started $($ Tau $=0.667, p=0.041$, SETau $=0.333)$. The study also included the implications for practitioners and researchers as the significant need to address multicultural competency in PFA is needed.

Keywords: psychological first aid, training, disaster behavioral health, diversity, COVID-19

\section{Introduction}

The COVID-19 pandemic has presented unique challenges since the World Health Organization (WHO) declared the pandemic caused by the SARS-CoV2 novel coronavirus (WHO, 2020). These unexpected public health crises highlight the need to manage stress and take care of one's mental health and wellbeing. Hence, psychological interventions are needed, mainly psychological first aid (PFA). With the COVID-19 related restrictions and physical distancing, remote PFA training and delivering is the safest method to meet the need for psychological aid during the global health crisis. Since COVID-19 started, there has been academic discourse related to online PFA training (Arenliu et al., 2020; Blake et al., 2020; Foster et al., 2019). Working remotely or in-person with the local community during the trauma is crucial to embed the culture competency interventions into the procedure. Marginalized and oppressed groups often have inequitable access to services
\end{abstract}


while impacted the most by disasters or health crises such as COVID-19 (Kantamneni, 2020; Shim \& Starks, 2021).

The goal of this study is twofold. The first goal is to help build the nomothetic net of PFA research. To do so, the authors of the present study probed the level of discourse on three important topics (race/ethnicity, general training delivery, online training delivery) in academic literature on PFA. The second goal was to conduct a study with potential to disrupt current practice less wise engage in research without usefulness to the PFA practitioner

A review of PFA research revealed four topics relevant to the variables included in this study. These topics were: (1) what is PFA, (2) what is known about PFA and diversity issues, (3) what is known about overall PFA training delivery, (4) what is known about online PFA training delivery, and (5) what is known about the relationship between the COVID-19 pandemic and intervention delivery. After these topics are discussed, the research questions that guided the present study will be detailed.

Psychological first aid is one of the vital tools in delivering psychological interventions to those who have undergone or experienced traumatic events. Understanding the structure of the psychological first aid research field allows for critical reflections and identification of blind spots for advancing the field. The term psychological first aid (PFA) first appeared in the military during World War II as a debriefing tool. Since then, PFA has become a robust implementation when working with acutely stressed individuals due to disaster or traumatic events. In the past, PFA was used to support individuals and the community's fear and anxiety during the outbreaks such as SARS and Ebola (WHO, 2014; Cénat, 2020) and by various responders such as nonprofessionals and mental health specialists (Fox et al., 2012). The multiple settings of PFA include faith-based organizations, health care facilities and nursing homes, K-12, workplaces, military, mass disaster venues, and in circumscribed critical incidents such as floods, fires, accidents, and other traumatic events (Allen et al., 2010; Cain et al., 2014; Hechanova et al., 2015). Since the COVID-19 pandemic started, there has been increased PFA support for frontline healthcare professionals and demonstrated a reduction in psychological distress (Asaoka et al., 2021; Muller et al., 2020).

PFA generally includes providing information, comfort, emotional care, and instrumental support to those experiencing disaster-related stress (Forbes et al., 2011; Fox et al., 2012). With the proliferation of PFA models, the effective delivery methods of PFA vary (Shultz \& Forbes, 2013). About a decade ago, a few systematic reviews discussed the lack of evidence-based data to develop guidelines for interventions and training procedures (Dieltjens et al., 2014; Fox et al., 2012; Shultz \& Forbes, 2014). To address the concerns for PFA's safety, effectiveness, and feasibility when used by nonprofessional implementers, Fox et al. (2012) systematically reviewed PFA evidence in 2000-2010. They concluded that there was insufficient evidence to support PFA treatment standard despite broad support by expert opinion. 
There are writings on PFA for healthcare workers (Corcoran, 2020), international settings (Yang et al., 2020), and specific demographic groups. In the Philippines, Hechanova et al. (2015) developed and evaluated a training program for disaster workers applying culturally sensitive and mindfulness-informed PFA intervention and found significant increases in knowledge about disaster reactions and coping self-efficacy. An international PFA program in Gaza was mobilized to support the local community due to wars (Schafer et al., 2016). Similarly, Malaysia adapted the remote PFA of the International Federation of Red Crescent Societies with phones and an online platform during COVID-19 (Sulaiman et al., 2020).

During the rapidly evolving protocols related to COVID-19, many mental health support programs or FPA prioritized frontline health care workers' clinical needs over research methods (Buselli et al., 2021). Only a handful of reviews and studies addressed diversity and structural racism issues when providing mental health support or PFA (Adler \& Bhattacharyya, 2021). Given the programs' heterogeneity, the lack of standardized protocols with a robust methodology, and the lack of clinical outcomes, the efficacy of comparisons among diverse groups was not available (Buselli et al., 2021).

PFA training was reported to significantly increase the providers' competency and selfefficacy required to respond appropriately after a traumatic event in a randomized controlled trial in Sierra Leone (Sijbrandij et al., 2020) and several non-randomized studies (Akoury-Dirani et al., 2015; Allen et al., 2010; Choi, 2020; Lalani \& Drolet, 2020; Lee et al., 2017; Lewis et al., 2014; Sijbrandij et al., 2020).

Mental health support or PFA delivery via digital platform has been developed or modified rapidly since COVID-19. These virtual approaches to support health care workers were presented globally, including China (Cheng et al., 2020; Yang et al., 2020), France (Alleaume et al., 2021), Malaysia (Sulaiman et al., 2020), U.K. (Blake et al., 2020), etc. For example, the simulation-based PFA training combined with the PLS mobile app improved the relief workers' performance in applying PFA in disaster situations (Kang \& Choi, 2021). However, it is an ongoing concern with minimal evidence-based psychological aid protocols to help health care workers cope with COVID-19 (Buselli et al., 2021).

This study aims to understand linguistic features and trends in psychological first aid programs. The study will examine abstracts with keywords of psychological first aid in academic peer-reviewed journals. Given those mentioned above, four research questions were developed to guide this study. These questions were:

RQ1: What are the usage rates of the term "psychological first aid" and the acronym "PFA"?

RQ2: How is the acronym "PFA" used in the academic discourse on psychological first aid?

RQ3: Within academic articles on "psychological first aid" from 1975 to 2021, what is the usage rate of terms related to race/ethnicity, learning delivery except for online, and online training delivery methods? 
RQ4: Within academic articles on "psychological first aid" from 2012 to 2021, did the usage rate of terms related to online training delivery methods change with the advent of the COVID-19 pandemic?

\section{Design}

\section{Method}

This study employed a cross-sectional observational design (Jepsen et al., 2004). The variables of research question four are time (pre-pandemic, pandemic) and online training delivery. The level of measurement is continuous. The unit of analysis was individual words. Given the public and published nature of the data, no human subjects review was required.

\section{Corpus}

\section{Register, Scope, and Sources}

The register was Academic English, and the sub-register was journal article abstracts. The scope was abstracts published in English for academic purposes between 1975 and 2021 that were produced using a keyword search of "psychological first aid" through the Web of Science database. This search yielded 255 abstracts that contained a total of 39,272 words and 5,075 word types. This corpus was divided into two sub-corpora for RQ4 (pre-pandemic v. pandemic). The pre-pandemic sub-corpus included abstracts published between January 1, 2012, and March 10, 2020. The pandemic subcorpus was composed of articles published between March 11, 2020, and December 31, 2021. The start date for the pandemic subcorpus was when the World Health Organization declared the start of the COVID-19 pandemic (WHO, 2020). The resultant sub-corpora varied in the abstract count and word count: (a) pre-pandemic: 164 abstracts and 19520 words; and (b) pandemic: 91 abstracts and 19752 words.

\section{Preprocessing}

The abstracts were downloaded from the Web of Science database in an Excel file. The text of the abstracts was then transformed into .txt files for analysis.

\section{Measures}

For this research project, the authors created three dictionaries (i.e., wordlists, lexicons). The dictionaries contained words, word stems, and bi-grams. A complete list of items in each dictionary can be found in Table 1.

\section{Race/Ethnicity Dictionary}

This dictionary contains 12 items. These items include the word race and the word stem multicult* ${ }^{*}$ This dictionary was drawn from a study of the academic discourse in play therapy (Page, 2021).

\section{Learning Delivery Except Online Dictionary}

This dictionary contains 19 items concerning learning delivery methods. These items include the word instruction and the word stem telecours*. 


\section{Online Learning Delivery Dictionary}

This dictionary contains 25 items of online learning delivery methods. These items include the word Zoom and the word stem synchronou*.

\section{Word Sketch}

Word sketches are a type of lexical profiling (Kilgarriff \& Rundell, 2003). This profiling includes how a word (i.e., node word) is used in concert with other words (i.e., collocations) and its grammatical features. Thus, a word sketch is a corpus-based summary of a word's grammatical and collocational behavior in graphical form (Kilgarriff \& Kosenm, 2012). The word sketch of the acronym PFA can provide the PFA research with some insight into the range of its use in academic English.

\section{Apparatus}

The authors conducted the linguistic analyses for R.Q.s 1 and 3 using \#Lancsbox 6.0 (Brezina et al., 2021). The word graph for RQ2 was produced using the linguistic analysis software program Sketch Engine (Kilgarriff et al., 2021). Differences in the use of online learning methods from the pre-pandemic period to the pandemic period (RQ4) were assessed using the single case research Tau calculator (Tarlow, 2016).

\section{Data Analysis}

Regarding RQ1, the raw and normalized counts for the term and the acronym will be reported. The normalized count will be the rate per 10,000 words. Concerning RQ2,_a figure showing the five words most associated with the following usages of PFA will be demonstrated by these grammar categories: (a) modifiers of PFA, (b) nouns modified by PFA, (c) verbs with PFA as object, and (d) verbs with PFA as the subject. Concerning RQ3, the raw word counts for all three dictionaries will be provided in tabular form. In addition, the raw and normalized counts (rate per 10,000) for all three dictionaries will be provided. Regarding RQ4, the difference between the pre-covid and covid periods was assessed using Tau (Tarlow, 2017). The alpha was set at $p<.05$, and all analyses took place using an online Tau calculator (Tarlow, 2016).

\section{Results}

Regarding RQ1, the obtained raw counts and normalized counts were: psychological first aid ( $\mathrm{raw}=201$, normalized $=51.18)$ and PFA (raw $=230$, normalized $=58.57)$. Concerning RQ2, the words and parts of grammar related to the acronym PFA can be inspected in Figure 1. PFA as a modifier has the most frequent appearances. In this category, the noun "training" was the word modified by the term "PFA". In terms of RQ3, the percentage of words in the dictionaries appearing in the corpus and the usage rates for the dictionaries can be reviewed in Table 2. About RQ4, the baseline trend Tau $=-0.043$, $p=1.000$, and thus no baseline correction was needed. Concerning the comparison of two phases (pre-pandemic v. pandemic), the alternative hypothesis was supported (Tau = $0.667, p=0.041, S E \mathrm{Tau}=0.333)$. The results are available for review in graphical form in Figure 2. 


\section{Discussion}

This study sought to explore the occurrence rate of "psychological first aid" and the acronym "PFA" used in the academic discourse. The study also examined the usage rate related to diversity, general training delivery methods, and online training delivery methods within the academic articles. The authors used Tau to analyze if the usage rate of terms related to online training delivery methods changed from 2012 to 2021 with the advent of the COVID-19 pandemic.

In terms of RQ1, two potential explanations for the obtained frequencies exist. First, using the trigram "Psychological First Aid" in a keyword search will pull in articles with the trigram and its associated acronym "PFA". Second, the exists in the academic discourse in the social science interest in "Psychological First Aid".

Regarding RQ2, two probable reasons exist for the obtained results on how the acronym PFA is used in the academic discourse on psychological first aid. The first explanation is that PFA represents variegated activities and processes and led to the acronym being used in a variety of ways, such as an adjective "PFA training" and as a noun "group-based PFA." An alternative explanation for the obtained results is that PFA is an emerging concept, and this fluidity leads to a multiplicity of word associations and grammatical functions. Between the latter and the former explanations, the former is most likely because of the long history of the use of the concept and the formal federal definition of the term in the U.S. (U.S. Department of Education, 2021).

In reference to RQ3, the race and diversity usage rate was only 6.11 per 10,000 counts. This low rate indicates that the existing PFA academic discourse did not prioritize diversity and equity. Marginalized and oppressed groups often have inequitable access to services while impacted the most by disasters or health crises such as COVID-19 (Kantamneni, 2020; Shim \& Starks, 2021). PFA is a relatively new set of techniques aimed to be deployed in traumatic events by emergency medical service workers who may or may not have advanced training in multicultural competency or self-efficacy to initiate racial equity discussion. An alternative explanation is that race, diversity, and equity are not relevant in the PFA procedure. The hierarchy of needs (Maslow, 1943) is universal across all cultures and races during traumatic events. Therefore, the priority is to address basic physiological needs, such as food and water. The latter is a less viable explanation as psychological first aid addresses the psychological reactions and needs. Hence, the increased multicultural competency is more likely to increase the rapport building with the local community during the aftermath of the trauma.

Concerning RQ4, this study has shown a significant increase in online PFA training since COVID-19. A similar result indicates increased demand for PFA deliveries digitally or via telephone (Arenliu et al. 2020). With the COVID-19 related restrictions and physical distancing, training and delivering PFA remotely is the safest method to meet the need for psychological aid during the global health crisis. The second probable reason is the increased funding supported by the government or organizations to develop online or remote PFA modules. For example, to protect and promote the psychological wellbeing of 
healthcare workers during the COVID-19 outbreak in the UK, Blake et al. (2020) developed a digital learning package using Agile methodology to support psychological wellbeing in healthcare works. A third likely explanation is the technological innovation through online platforms. This advancement along the low costs of development and maintenance allows for e-delivering and e-learning PFA efficiently (Blake et al., 2020; Foster et al., 2019), especially brief online intervention modules to enhance caretakers' resilience psychological wellbeing (Foster et al., 2019). The increase in online delivery is likely because of the combination of all three of the above reasons.

When interpreting the results, one should consider the following limitations. First, the corpus size was limited despite all the available academic publications being included. This study only examined the published English academic abstracts from the database Web of Science. A larger corpus with increased publication number and more words from the full articles will create greater word frequency and more detailed collocation graphs. Second, only keyword "psychological first aid" was used in the search. Different authors may loosely define this phrase, and therefore the PFA delivery contents, curriculum, and models vary in the search results. For example, the related phrase "mental health first aid" could yield different results. It has a different purpose with different procedures, yet elements such as rapport building often overlap with PFA when offering the initial psychological support. Future studies can consider the extent of the contents available in PFA publications. Despite these limitations, there are valuable implications for clinical practice and research related to psychological first aid.

This study indicates a significant need to increase multicultural competency in PFA training and service delivery as race discussions often are not recognized. Multicultural competency and awareness about systemic oppression, racial identity, white privilege, and microaggression are often complex and taboo topics. This further presents the crucial role trainers play to include the aforementioned through the social justice lens lest they negatively impact rapport and service delivery efficacy (Sue et al., 2019).

Existing publications focused on service delivery, less on training, and even fewer on online training methods. As first responder organizations increasingly adopt evidence-informed interventions, researchers should conduct formative evaluations to check for training drift. Future research should examine the variables that promote the PFA delivery effectiveness at the more granular level. This leads to further implications for the PFA studies to explore the multicultural competency of both the trainers and trainees and an effective curriculum to increase the first responders' self-efficacy in the race and equity discussions.

\section{References}

Adler, S. \& Bhattacharyya, S. (2021). Beyond the nurses and doctors: Structural racism and the unseen frontline service workers during the COVID-19 pandemic. Psychiatric Services, $\quad$ 72(5), 594-596. https://doi.org/10.1176/appi.ps.202000569

Akoury-Dirani, L., Sahakian, T. S., Hassan, F. Y., Hajjar, R. V., \& Asmar, K. E. (2015). Psychological first aid training for Lebanese field workers in the emergency context 
of the Syrian refugees in Lebanon. Psychological Trauma: Theory, Research, Practice, and Policy, 7(6), 533-538. https://doi.org/10.1037/tra0000028

Alleaume, C., Verger, P., \& Peretti-Watel, P. (2021). Psychological support in general population during the COVID-19 lockdown in France: Needs and access. PloS One, 16(5), e0251707-e0251707. https://doi.org/10.1371/journal.pone.0251707

Allen, B., Brymer, M. J., Steinberg, A. M., Vernberg, E. M., Jacobs, A., Speier, A. H., \& Pynoos, R. S. (2010), Perceptions of psychological first aid among providers responding to Hurricanes Gustav and Ike. Journal of Traumatic Stress, 23(4), 509513. https://doi.org/10.1002/jts.20539

Arenliu, A., Uka, F., \& Weine, S. (2020). Building online and telephone psychological first aid services in a low resource setting during COVID-19: the case of Kosovo. Psychiatria Danubina, 32(3-4), 570-576. https://doi.org/10.24869/psyd.2020.570

Asaoka, H., Koido, Y., Kawashima, Y., Ikeda, M., Miyamoto, Y., \& Nishi, D. (2021). Longitudinal change of psychological distress among healthcare professionals with and without psychological first aid training experience during the COVID-19 pandemic. International Journal of Environmental Research and Public Health, 18(23), 12474. https://doi.org/10.3390/ijerph182312474

Blake, H., Bermingham, F., Johnson, G., \& Tabner, A. (2020). Mitigating the Psychological Impact of COVID-19 on Healthcare Workers: A Digital Learning Package. International Journal of Environmental Research and Public Health, 17(9), 2997. https://doi.org/10.3390/ijerph17092997

Brezina, V., Weill-Tessier, P., \& McEnery, A. (2021). \#LancsBox v. 6.x. [software package]. http://corpora.lancs.ac.uk/lancsbox/

Buselli, R., Corsi, M., Veltri, A., Baldanzi, S., Chiumiento, M., Del Lupo, E., Marino, R., Necciari, G., Caldi, F., Foddis, R., Guglielmi, G., \& Cristaudo, A. (2021). Mental health of Health Care Workers (HCWs): a review of organizational interventions put in place by local institutions to cope with new psychosocial challenges resulting from COVID-19. Psychiatry Research, 299, 113847-113847. https://doi.org/10.1016/j.psychres.2021.113847

Cain, Plummer, C. A., Fisher, R. M., \& Bankston, T. Q. (2014). Weathering the storm: Persistent effects and psychological first aid with children displaced by hurricane Katrina. Journal of Child \& Adolescent Trauma, 3(4), 330-343. https://doi.org/10.1080/19361521.2010.523063

Cénat, Mukunzi, J. N., Noorishad, P.-G., Rousseau, C., Derivois, D., \& Bukaka, J. (2020). A systematic review of mental health programs among populations affected by the Ebola virus disease. Journal of Psychosomatic Research, 131, 109966-109966. https://doi.org/10.1016/j.jpsychores.2020.109966

Cheng, Z. F., Hua, Y., Yang, Z., \& Liu, J. (2020). Development of a psychological first-aid model in inpatients with COVID-19 in Wuhan, China. General Psychiatry, 33(3), e100292-e100292. https://doi.org/10.1136/gpsych-2020-100292

Choi, Y. J. (2020). Psychological First-Aid Experiences of Disaster Health Care Workers: A Qualitative Analysis. Disaster medicine and public health preparedness, 14(4), 433-436. https://doi.org/10.1017/dmp.2019.87 
Cohen, J. (1988). Statistical power analysis for the social sciences ( $2^{\text {nd }}$ ed.). Lawrence Erlbaum Associates.

Corcoran, C. (2020). Use of psychological first aid for nurses. Nursing Economics, 38 (1), 26-32.

Dieltjens, T., Moonens, I., Van Praet, K., De Buck, E., \& Vandekerckhove, P. (2014). A systematic literature search on psychological first aid: Lack of evidence to develop $\begin{array}{lllll}\text { guidelines. } & P L O S & \text { ONE, } & 9(12), & \text { e114714. }\end{array}$ https://doi.org/10.1371/journal.pone.0114714

Faul, F., Erdfelder, E., Buchner, A., \& Lang, A. (2009). Statistical power analyses using G*Power 3.1: Tests for correlation and regression analyses. Behavior Research Methods, 41(4), 1149-1160. https://doi.org/10.3758/brm.41.4.1149.

Foster, K., Mitchell, R., Young, A., Van, C., \& Curtis, K. (2019). Resilience-promoting factors for parents of severely injured children during the acute hospitalisation period: A qualitative inquiry. Injury, 50(5), 1075-1081. https://doi.org/10.1016/j.injury.2018.12.011

Fox, J., Burkle, F., Bass, J., Pia, F., Epstein, J., \& Markenson, D. (2012). The effectiveness of psychological first aid as a disaster intervention tool: Research analysis of peerreviewed literature from 1990-2010. Disaster Medicine and Public Health Preparedness, 6(3), 247-252. https://doi.org/10.1001/dmp.2012.39

Hechanova, R. M., Ramos, P. A. P. \& Waelde, L. (2015). Group-based mindfulnessinformed psychological first aid after Typhoon Haiyan. Disaster Prevention and Management, Vol. 24 No. 5, pp. 610-618. https://doi.org/10.1108/DPM-01-20150015

Kang, J.-Y., \& Choi, Y.-J. (2021). Effects of a psychological first aid simulated training for pregnant flood victims on disaster relief worker's knowledge, competence, and selfefficacy. Applied Nursing Research, 57, 151348, https://doi.org/10.1016/j.apnr.2020.151348

Kantamneni N. (2020). The impact of the COVID-19 pandemic on marginalized populations in the United States: A research agenda. Journal of Vocational Behavior, 119, 103439. https://doi.org/10.1016/j.jvb.2020.103439

Kilgarriff, A., \& Kosem, I. (2012). Corpus tools for lexicographers. In S. Granger \& M. Paquot (Eds.), Electronic Lexicography (pp. 31-56). OUP Oxford. 10.1093/acprof:oso/9780199654864.003.0003

Kilgarriff, A., \& Rundell, M. (2003). Lexical profiling software and its lexicographic applications: a case study. In A. Braasch and C. Povlsen (Eds.), Proceedings of the Tenth EURALEX International Congress, EURALEX 2002 (pp 807-818). Center for Sprogteknologi. https://macmillandictionaries.com/MEDMagazine/November2002/02-Word-Sketches.htm

Kilgarriff, A., Rychlý, P., Smrž, P., \& Tugwell, D. (2021). The Sketch Engine [software]. http://www.sketchengine.eu/ Technology.

Lalani, N. S., \& Drolet, J. (2020). Effectiveness of psychological first aid training for social work students, practitioners and human service professionals in Alberta, Canada. The Journal of Practice Teaching and Learning, 17, 7-21. https://doi.org/10.1921/jpts.v17i1.1269 
Lee, J.-S., You, S., Choi, Y.-K., Youn, H.-Y., \& Hye, S. S. (2017). A preliminary evaluation of the training effects of a didactic and simulation-based psychological first aid program in students and school counselors in South Korea. PLoS One, 12(7), e0181271. https://doi.org/10.1371/journal.pone.0181271

Lewis, V., Varker, T., Phelps, A., Gavel, E., \& Forbes, D. (2014). Organizational implementation of psychological first aid (PFA): Training for managers and peers. Psychological Trauma: Theory, Research, Practice and Policy, 6(6), 619-623. https://doi.org/10.1037/a0032556

Maslow, A. H. (1943). A theory of human motivation. Psychological Review, 50(4), 370396. https://doi.org/10.1037/h0054346

Muller, A. E., Hafstad, E. V., Himmels, J. P. W., Smedslund, G., Flottorp, S., Stensland, S., Stroobants, S., Van de Velde, S., \& Vist, G. E. (2020). The mental health impact of the covid-19 pandemic on healthcare workers, and interventions to help them: A rapid systematic review. Psychiatry Research, 293, 113441. https://doi.org/10.1016/j.psychres.2020.113441

Page, U. (2021). Page race ethnicity dictionary in REGEX. OSF Research Project [website]. https://osf.io/f2gyq/

Ruzek, J. I., Brymer, M. J., Jacobs, A. K., Layne, C. M., Vernberg, E. M., \& Watson, P. J. (2007). Psychological First Aid. Journal of Mental Health Counseling, 29 (1), 17-49. https://doi.org/10.17744/mehc.29.1.5racqxjueafabgwp

Schafer, A. Snider, L., Sammour, R., \& Sammour, R. (2016). A reflective learning report about the implementation and impacts of Psychological First Aid (PFA) in Gaza. Disaster Health, 3(1), 1-10. https://doi.org/10.1080/21665044.2015.1110292

Shim, R. S. \& Starks, S. M. (2021). COVID-19, Structural racism, and mental health inequities: Policy implications for an emerging syndemic. Psychiatric Services, 72(10), 1193-1198. https://doi.org/10.1176/appi.ps.202000725

Sijbrandij, M., Horn, R., Esliker, R., O'May, F., Reiffers, R., Ruttenberg, L., Stam, K., de Jong, J. \& Ager, A. (2020). The effect of Psychological First Aid Training on knowledge and understanding about psychosocial support principles: A clusterrandomized controlled trial. International Journal of Environmental Research and Public Health, 17(2), 484. https://doi.org/10.3390/ijerph17020484

Sokolowski, H. M., Hawes, Z., \& Lyons, I. M. (2019). What explains sex differences in math anxiety? A closer look at the role of spatial processing. Cognition, 182, 193-212. https://doi.org/10.1016/j.cognition.2018.10.005

Sue, D. W., Sue, D., Neville, H. \& Smith, L. (2019). Counseling the culturally diverse: Theory and practice ( $8^{\text {th }}$ ed.). J. Wiley.

Sulaiman, A. S., Z., Jaafa, M. J., Francis, B., Razali, K. A., Juares Rizal, A., Mokhtar, N. H., Juhari, J. A., Zainal, S., \& Ng, C. G. (2020). Development of a Remote Psychological First Aid Protocol for Healthcare Workers Following the COVID-19 Pandemic in a University Teaching Hospital, Malaysia. Healthcare, 8(3), 228. https://doi.org/10.3390/healthcare8030228

Tarlow, K. R. (2016). Baseline Corrected Tau Calculator \{Software]. http://www.ktarlow.com/stats/tau 
Tarlow, K. R. (2017). An improved rank correlation effect size statistic for single-case designs: Baseline Corrected Tau. Behavior Modification, 41(4), 427-467. http://dx.doi.org/10.1177/0145445516676750

Tessier, M., Lamothe, J., \& Geoffrion, S. (2021). Adherence to Psychological First Aid after Exposure to a Traumatic Event at Work among EMS Workers: A Qualitative Study. International Journal of Environmental Research and Public Health, 18(21), 11026. https://doi.org/10.3390/ijerph182111026

U.S. Department of Education. (2021). 10 tips for teaching the Psychological First Aid Model for $\quad \mathrm{K}-12$ education agencies. https://rems.ed.gov/Docs/PFA_10_Tips_508C.pdf

World Health Organization, CBM, World Vision International and UNICEF (2014). Psychological First aid During Ebola Virus Disease Outbreaks. https://apps.who.int/iris/bitstream/handle/10665/131682/9789241548847_eng.p df

World Health Organization, WHO. (2020). Timeline: WHO's COVID-19 response. https://www.who.int/emergencies/diseases/novel-coronavirus-2019/interactivetimeline

Yang, J., Tong, J., Meng, F., Feng, Q., Ma, H., Shi, C., Yuan, J., Yang, S., Liu, L., Xu, L., Xi, Y.-J., Li, W., Rohlof, H., Zhao, X. \& Kang, C. (2020). Characteristics and challenges of psychological first aid in China during the COVID-19 outbreak. Brain, Behavior, and Immunity, 87, 113-114. https://doi.org/10.1016/j.bbi.2020.04.075 
Table 1

Results from Dictionaries (RQ4)

\begin{tabular}{|c|c|c|c|}
\hline Word & Gen. Raw Ct & Online Raw Ct. & R/E Raw Count \\
\hline "of color" & & & 0 \\
\hline *-cult* & & & 0 \\
\hline *cast & & 0 & \\
\hline anytime-anywhere & 0 & & \\
\hline asynchronou* & & 0 & \\
\hline audioconferenc* & & 0 & \\
\hline bias & & & 0 \\
\hline blackboard & & 0 & \\
\hline blended & & 0 & \\
\hline canvas & & 0 & \\
\hline competenc* & 17 & & \\
\hline compute* & 0 & & \\
\hline courseware & 0 & & \\
\hline cult* & & & 21 \\
\hline digital & & 6 & \\
\hline distance & 1 & & \\
\hline divers* & & & 1 \\
\hline e-* & & 4 & \\
\hline elearning & & 0 & \\
\hline ethnic* & & & 0 \\
\hline $\mathrm{f} 2 \mathrm{f}$ & 0 & & \\
\hline face-to-face & 0 & & \\
\hline google meet & & 0 & \\
\hline gotomeeting & & 0 & \\
\hline hybrid & & 0 & \\
\hline in-person & 0 & & \\
\hline instruction & 0 & & \\
\hline interne* & & 1 & \\
\hline learning & 11 & & \\
\hline marginali* & & & 0 \\
\hline media & 2 & & \\
\hline microsoft teams & & 0 & \\
\hline minorit* & & & 0 \\
\hline moodle & & 0 & \\
\hline multicult* & & & 1 \\
\hline
\end{tabular}




\begin{tabular}{lccc}
$\begin{array}{l}\text { multimedia } \\
\text { online }\end{array}$ & 0 & 4 & 1 \\
prejudice & & & 0 \\
pretrain* & 1 & & \\
race & & & \\
screencas* & 0 & & \\
self dir* & 31 & & \\
self-* & & 0 & \\
stereotyp* & 0 & \\
streami* & & & \\
synchronou* & & & \\
teleconferenc* & 0 & & \\
telecours* & 0 & 0 & \\
train* & 188 & & \\
threaded & & 1 & \\
untrain* & 3 & 5 & \\
video & & 0 & \\
videoconferenc* & & & \\
virtual & & & \\
web* & & & \\
zoom & & & \\
\hline
\end{tabular}


Table 2

Dictionary Counts (RQ3)

\begin{tabular}{lccccc}
\hline Dictionary & $\begin{array}{l}\text { Words in } \\
\text { Dictionary }\end{array}$ & $\begin{array}{l}\text { Words in } \\
\text { Dictionary } \\
\text { Present in } \\
\text { Corpus }\end{array}$ & $\begin{array}{l}\text { \% of Words in } \\
\text { Dictionary } \\
\text { Present in } \\
\text { Corpus }\end{array}$ & $\begin{array}{l}\text { Dictionary } \\
\text { Raw Word } \\
\text { Count }\end{array}$ & $\begin{array}{l}\text { Dictionary } \\
\text { Normalized } \\
\text { Word Count } \\
\text { (per 10,000) }\end{array}$ \\
\hline Race/Ethnicity & 12 & 4 & $33 \%$ & 24 & 6.11 \\
$\begin{array}{l}\text { Learning Delivery } \\
\text { Except Online }\end{array}$ & 19 & 8 & $42 \%$ & 251 & 63.91 \\
$\begin{array}{l}\text { Online Learning } \\
\text { Delivery }\end{array}$ & 25 & 7 & $28 \%$ & 22 & 5.60 \\
\hline
\end{tabular}

Note. $n=39,272$. 
Figure 1

Usage of the PFA Acronym (RQ2)

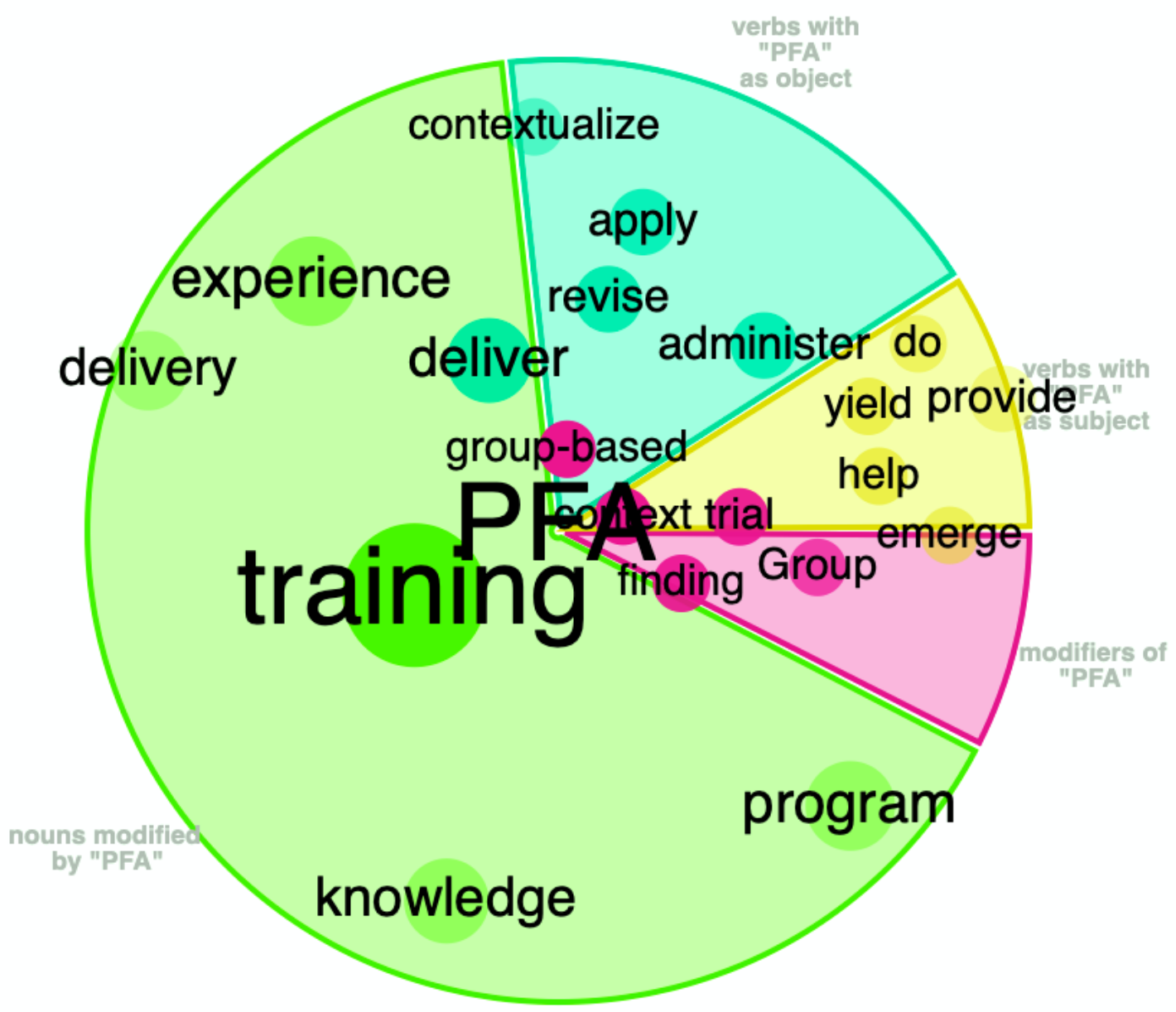

Note. Distance from the center denotes typicality ("PFA Training" is more typical than "PFA

Knowledge". The circle's size indicates frequency. Circle color indicates which grammar category the word belongs to, although sometimes a word occurs appears outside of its category to visualize other statistics accurately. Category size indicates the size of that category in relation to other graphical categories. This description is based on Word Sketch module explanation appearing in the Sketch Engine software (Kilgarriff et al., 2021). 
Figure 2

Covid Pre-Pandemic to Pandemic Online Word Use Shift (RQ3)

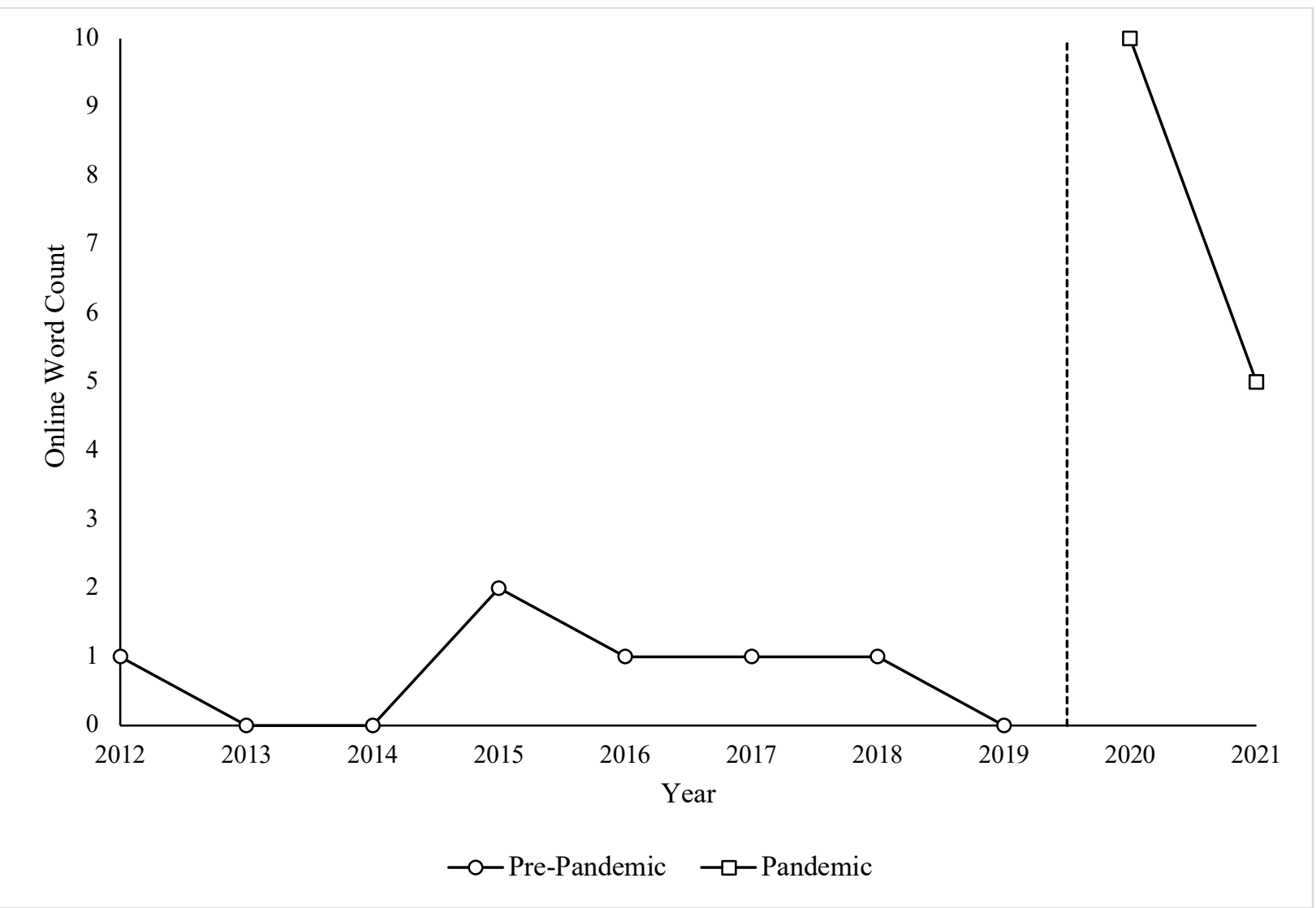

